\title{
Coupled-mode Analysis of Plasmonic MIM Waveguide Coupled with a Resonant Cavity
}

\author{
Tomotaka Ikeda $^{1}$, Toshiaki Kitamura ${ }^{1, *}$, Kiyoshi Kishioka ${ }^{2}$ \\ ${ }^{1}$ Faculty of Engineering Science, Kansai University, Japan \\ ${ }^{2}$ Faculty of Engineering, Osaka Electro-Communication University, Japan
}

Copyright (C) 2015 by authors, all rights reserved. Authors agree that this article remains permanently open access under the terms of the Creative Commons Attribution License 4.0 International License.

\begin{abstract}
The coupling coefficients of two parallel metal-insulator-metal (MIM) plasmonic waveguides are investigated using the analysis of the coupled-mode equations. The frequency characteristics of the power transmittance of a MIM waveguide coupled with a resonant cavity are studied and compared with the simulation results obtained from the FDTD method into which motion equations of free electrons are installed.
\end{abstract}

Keywords Metal-insulator-metal Waveguide, Surface Plasmon Polariton, Coupled Mode Analysis, FDTD Method

\section{Introduction}

Electromagnetic waves that propagate along the metal-dielectric interface, known as surface plasmon polaritons (SPPs), have attracted much interest due to their potential to realize nanoscale photonic devices with the dimension smaller than the diffraction limit. Mainly two types of configurations, metal-insulator-metal (MIM) and insulator -metal-insulator (IMI), have been utilized for the planar SPP devices, and especially, MIM configurations have been attracting much attention because they have advantages due to their strong field localization, simplicity, and easiness of fabrication. Various MIM plasmonic devices have been proposed, such as Bragg gratings [1], ring resonators [2-4], Splitters [5,6], and nanodisk cavities [7]. Stubs and resonators using rectangular cavities have also been studied [8-13]. Most of these studies have utilized FDTD simulation to investigate the characteristics.

In this study, we deal with a MIM resonant cavity coupled with a MIM waveguide by using the coupled-mode theory $[14,15]$ which can analyze the characteristics while saving the computer resources. The coupling coefficients of two parallel MIM waveguides are analyzed utilizing the coupled-mode equations. The frequency characteristics of the power transmittance of a MIM waveguide are studied and compared with the simulation results. The simulations are carried out by using the FDTD method into which motion equations of free electrons are installed [16].

\section{Metal-Insulator-Metal Waveguide}

Figure 1 shows a MIM waveguide coupled with a resonant cavity. Here, it is assumed that the cavity has the same width (2a) as the waveguide and the length along the propagation direction is L. First, we describe the fundamental propagation modes of a MIM waveguide which consists of silver and air.

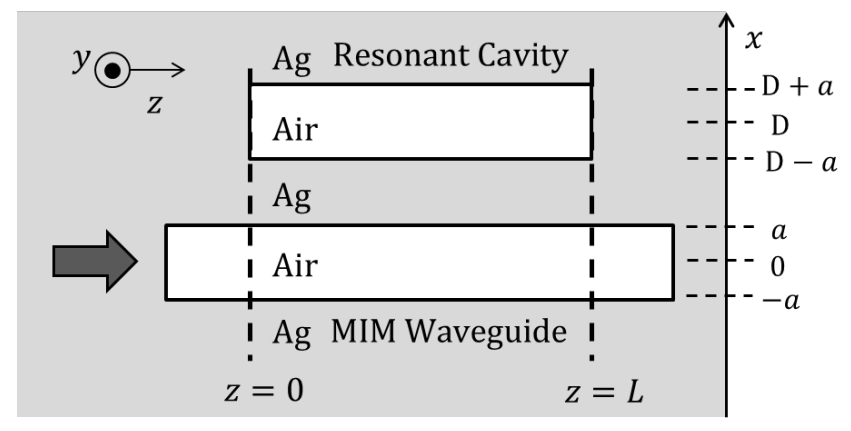

Figure 1. MIM waveguide coupled with a resonant cavity

In this study, we deal with silver as ideal metal which has the dielectric function of the undamped free electron plasma. The relative permittivity of the silver is expressed using the Drude model as follows:

$$
\begin{gathered}
\varepsilon_{r}(\omega)=1-\frac{\omega_{p}{ }^{2}}{\omega^{2}+j \omega \gamma} \\
=\left(1-\frac{\omega_{p}^{2}}{\omega^{2}+\gamma^{2}}\right)+j\left(\frac{\omega_{p}^{2} \gamma}{\omega^{3}+\omega \gamma^{2}}\right)
\end{gathered}
$$

where $\omega_{p}$ and $\gamma$ are the plasma frequency and the collision frequency, respectively. Here, we assume the lossless ideal metal so that $\gamma=0$ and $\omega_{p}=9.1[\mathrm{eV}]$.

When we consider the fundamental transverse-magnetic (TM) mode of the MIM waveguide, the electromagnetic fields of each region are described by the following equations:

$$
\left\{\begin{array}{l}
H_{y}=\mathrm{A} e^{-k_{2} x} e^{-j \beta z} \\
E_{x}=\mathrm{A} \frac{\beta}{\omega \varepsilon_{0} \varepsilon_{2}} e^{-k_{2} x} e^{-j \beta z} \\
E_{z}=j \mathrm{~A} \frac{k_{2}}{\omega \varepsilon_{0} \varepsilon_{2}} e^{-k_{2} x} e^{-j \beta z}
\end{array}\right.
$$

for $x>a$ and 


$$
\left\{\begin{array}{l}
H_{y}=\mathrm{A} e^{k_{2} x} e^{-j \beta z} \\
E_{x}=\mathrm{A} \frac{\beta}{\omega \varepsilon_{0} \varepsilon_{2}} e^{k_{2} x} e^{-j \beta z} \\
E_{z}=-j \mathrm{~A} \frac{k_{2}}{\omega \varepsilon_{0} \varepsilon_{2}} e^{k_{2} x} e^{-j \beta z}
\end{array}\right.
$$

for $x<-a$, and

$$
\left\{\begin{aligned}
& H_{y}= \mathrm{B}\left(e^{k_{1} x}+e^{-k_{1} x}\right) e^{-j \beta z} \\
& E_{x}= \mathrm{B} \frac{\beta}{\omega \varepsilon_{0} \varepsilon_{1}}\left(e^{k_{1} x}+e^{-k_{1} x}\right) e^{-j \beta z} \\
& E_{z}=-j \mathrm{~B} \frac{k_{1}}{\omega \varepsilon_{0} \varepsilon_{1}}\left(e^{k_{1} x}-e^{-k_{1} x}\right) e^{-j \beta z} \\
& \text { for }-a<x<a,
\end{aligned}\right.
$$

where $k_{i}$ and $\varepsilon_{i}(i=1,2)$ represent the wavenumbers along the $x$ axis and the relative permittivity, respectively, of each region.

\section{Coupling Coefficients}

The coupling coefficient of the two parallel MIM waveguides is calculated by the following equation [17]:

$$
c=\frac{\omega \varepsilon_{0}\left(\varepsilon_{2}-\varepsilon_{1}\right) \int_{-a}^{a} \boldsymbol{E}_{\mathbf{1}}{ }^{*} \cdot \boldsymbol{E}_{\mathbf{2}} d x}{\int_{-\infty}^{\infty} \boldsymbol{u}_{\mathrm{z}} \cdot\left(\boldsymbol{E}_{\mathbf{1}}{ }^{*} \times \boldsymbol{H}_{\mathbf{1}}+\boldsymbol{E}_{\mathbf{1}} \times \boldsymbol{H}_{\mathbf{1}}{ }^{*}\right) d x}
$$

Equation (5) is evaluated using the field components of TM mode. The electric fields are related to the magnetic fields as $E_{x}=\left(\beta / \omega \varepsilon_{0} \varepsilon_{i}\right) H_{y}$ and $E_{z}=\left(1 / \omega \varepsilon_{0} \varepsilon_{i}\right) \partial H_{y} /$ $\partial x(i=1,2)$. First, we consider the numerator of (5). The electric fields in each region are described by the following equations:

$$
\left\{\begin{array}{l}
x>a, x<-a: E_{1 x}=\frac{\beta}{\omega \varepsilon_{0} \varepsilon_{2}} H_{1 y} \\
-a<x<a: E_{1 x}=\frac{\beta}{\omega \varepsilon_{0}} H_{1 y}
\end{array}\right.
$$

for $E_{1 x}, H_{1 y}$, and

$$
\left\{\begin{array}{l}
x>\mathrm{D}+a, x<\mathrm{D}-a: E_{2 x}=\frac{\beta}{\omega \varepsilon_{0} \varepsilon_{2}} H_{2 y} \\
\mathrm{D}-a<x<\mathrm{D}+a: E_{2 x}=\frac{\beta}{\omega \varepsilon_{0}} H_{2 y}
\end{array}\right.
$$

for $E_{2 x}, H_{2 y}$, respectively.

Therefore, the scalar product of the electric fields is described using the magnetic fields by the following equation:

$$
\boldsymbol{E}_{1}^{*} \cdot \boldsymbol{E}_{2}=E_{1 x}{ }^{*} E_{2 x}+E_{1 z}{ }^{*} E_{2 z}
$$

for the denominator.

$$
=\frac{1}{\omega^{2} \varepsilon_{0}^{2} \varepsilon_{2}}\left(\beta^{2} H_{1 y}{ }^{*} H_{2 y}+\frac{\partial H_{1 y}{ }^{*}}{\partial x} \frac{\partial H_{2 y}}{\partial x}\right)
$$

Next, we consider the denominator of (5). Similarly, the vector product of electric and magnetic fields is expressed as

$$
\boldsymbol{u}_{z} \cdot\left(\boldsymbol{E}_{1}{ }^{*} \times \boldsymbol{H}_{1}+\boldsymbol{E}_{1} \times \boldsymbol{H}_{1}{ }^{*}\right)=\frac{2 \beta}{\omega \varepsilon_{0} \varepsilon}\left|H_{1 y}\right|^{2}
$$

where, $i=1$ for $-a<x<a$, and $i=2$ for $x>a$ or $x<-a$. Thus, the coupling coefficient is expressed using the magnetic fields as follows:

$$
\frac{\frac{1-\varepsilon_{2}}{\varepsilon_{2}}\left(\beta^{2} \int_{-a}^{a} H_{1 y}{ }^{*} H_{2 y} d x+\int_{-a}^{a} \frac{\partial H_{1} y^{*} \partial H_{2 y}}{\partial x} d x\right)}{2 \beta \int_{-\infty}^{\infty} \frac{1}{\varepsilon}\left|H_{1 y}\right|^{2} d x}
$$

Here, we set the relative permittivity in the air region as $\varepsilon_{1}=1$. The constant B of (4) is related to the constant A as follow:

$$
\mathrm{B}=\mathrm{A} e^{-k_{1} a-k_{2} a}\left(k_{1} \varepsilon_{2}-k_{2}\right) / 2 k_{1} \varepsilon_{2}
$$

And also, $H_{2 y}$ is written as $H_{2 y}=\mathrm{A} e^{k_{2}(x-\mathrm{D})} e^{-j \beta z}$. Therefore, the integrals in (10) are calculated as follows:

$$
\begin{gathered}
\int_{-a}^{a} H_{1 y}{ }^{*} H_{2 y} d x=2 \mathrm{AB} \int_{-a}^{a} e^{k_{2}(x-\mathrm{D})} \cosh k_{1} x d x \\
=\frac{2 \mathrm{~A}^{2}\left(k_{1} \varepsilon_{2}-k_{2}\right)}{\left(k_{1}{ }^{2}-k_{2}{ }^{2}\right) \varepsilon_{2}} e^{-k_{1} a-k_{2} a-k_{2} \mathrm{D}} \\
\times\left(\sinh k_{1} a \cosh k_{2} a-\frac{k_{2}}{k_{1}} \cosh k_{1} a \sinh k_{2} a\right)
\end{gathered}
$$

And

$$
\begin{gathered}
\int_{-a}^{a} \frac{\partial H_{1 y}{ }^{*}}{\partial x} \cdot \frac{\partial H_{2 y}}{\partial x} d x \\
=\mathrm{A}^{2} k_{1} k_{2} e^{-k_{1} a-k_{2} a} \frac{k_{1} \varepsilon_{2}-k_{2}}{k_{1} \varepsilon_{2}} \int_{-a}^{a} e^{k_{2}(x-D)} \sinh k_{1} x d x \\
=\frac{2 \mathrm{~A}^{2} k_{1} k_{2}\left(k_{1} \varepsilon_{2}-k_{2}\right)}{\left(k_{1}{ }^{2}-k_{2}{ }^{2}\right) \varepsilon_{2}} e^{-k_{1} a-k_{2} a-k_{2} \mathrm{D}} \\
\times\left(\cosh k_{1} a \sinh k_{2} a-\frac{k_{2}}{k_{1}} \sinh k_{1} a \cosh k_{2} a\right)
\end{gathered}
$$

for the numerator, and

$$
\begin{aligned}
\int_{-\infty}^{\infty} \frac{1}{\varepsilon}\left|H_{1 y}\right|^{2} d x= & \frac{\mathrm{A}^{2}}{\varepsilon_{2}} \int_{-\infty}^{-a} e^{2 k_{2} x} d x+4 \mathrm{~B}^{2} \int_{-a}^{a} \cosh ^{2} k_{1} x d x \\
& +\frac{\mathrm{A}^{2}}{\varepsilon_{2}} \int_{a}^{\infty} e^{-2 k_{2} x} d x \\
=\frac{\mathrm{A}^{2}}{k_{2} \varepsilon_{2}} e^{-2 k_{2} a}+ & \frac{\mathrm{A}^{2}}{2 k_{1}} e^{-2 k_{1} a-2 k_{2} a} \frac{\left(k_{1} \varepsilon_{2}-k_{2}\right)^{2}}{k_{1}{ }^{2} \varepsilon_{2}{ }^{2}}\left(\sinh 2 k_{1} a\right. \\
& \left.+2 k_{1} a\right) \\
= & \frac{\mathrm{A}^{2}}{\varepsilon_{2}} e^{-2 k_{2} a}\left\{\frac{1}{k_{2}}+\frac{e^{-2 k_{1} a}\left(k_{1} \varepsilon_{2}-k_{2}\right)^{2}}{2 k_{1}{ }^{3} \varepsilon_{2}}\left(\sinh 2 k_{1} a+2 k_{1} a\right)\right\}
\end{aligned}
$$

As a result, the coupling coefficient $c$ is written as

$$
c=\frac{\left(1-\varepsilon_{2}\right) k_{1}{ }^{2} k_{2}\left(k_{1} \varepsilon_{2}-k_{2}\right) e^{-k_{1} a+k_{2} a-k_{2} \mathrm{D}}\left\{k_{1}\left(\beta^{2}-k_{2}{ }^{2}\right) \sinh k_{1} a \cosh k_{2} a+k_{2}\left(k_{1}{ }^{2}-\beta^{2}\right) \cosh k_{1} a \sinh k_{2} a\right\}}{\beta\left(k_{1}{ }^{2}-k_{2}{ }^{2}\right)\left\{k_{1}{ }^{3} \varepsilon_{2}+k_{2}\left(k_{1} \varepsilon_{2}-k_{2}\right)^{2} e^{-2 k_{1} a}\left(\sinh k_{1} a \cosh k_{1} a+k_{1} a\right)\right\}}
$$




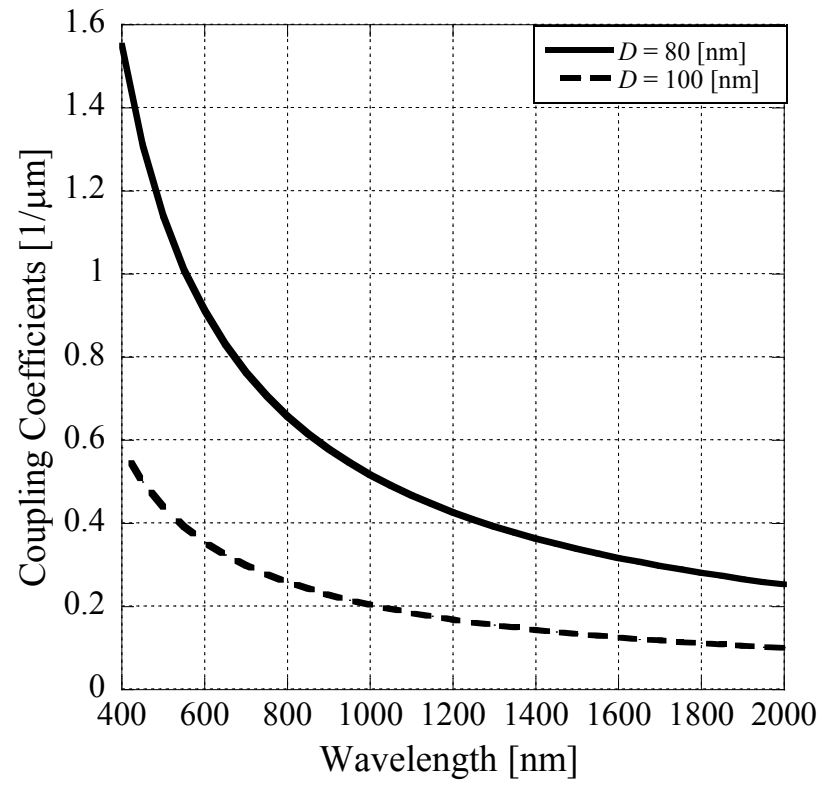

Figure 2. Coupling coefficients as a function of incident wavelength

Figure 2 shows the coupling coefficients as a function of the incident wavelength. Here, the half width of the waveguides $a=25[\mathrm{~nm}]$. We have confirmed that the coupling coefficients coincide with the ones which are obtained from the propagation constants of even and odd modes [17].

\section{Characteristics of Transmittance}

Figure 3 shows field amplitudes at the boundaries. When considering the forward wave, the field amplitude $\boldsymbol{a}^{+}(z)(z>0)$ is expressed by the transfer matrix $\hat{\mathbf{F}}(\mathrm{z})$ and the field amplitude at the input port $\boldsymbol{a}^{+}(0)$ as follows [15]:

$$
\boldsymbol{a}^{+}(z)=e^{-j \beta z} \hat{\mathbf{F}}(z) \boldsymbol{a}^{+}(0)
$$

The components of the transfer matrix $f_{i j}(i, j=1,2)$ are defined by using the coupling coefficient as $f_{11}(z)=$ $f_{22}(z)=\cos c z, f_{12}(z)=f_{21}(z)=-j \sin c z$. Similarly, the backward waves are expressed by using the transfer matrix. Therefore, the field amplitudes of the forward and backward waves at $z=0$ and $L$ are related to each other as

$$
\begin{aligned}
\boldsymbol{a}^{+}(L) & =e^{-j \beta L} \hat{\mathbf{F}}(L) \boldsymbol{a}^{+}(0) \\
\boldsymbol{a}^{-}(0) & =e^{-j \beta L} \hat{\mathbf{F}}(L) \boldsymbol{a}^{-}(L)
\end{aligned}
$$

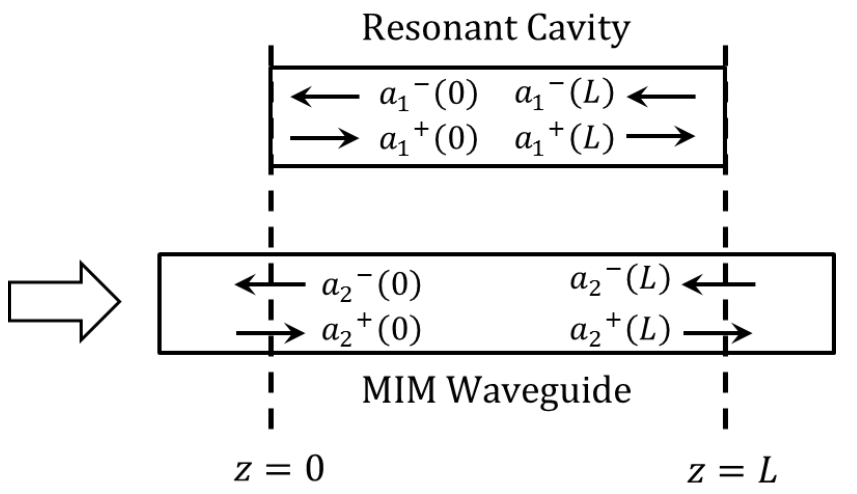

Figure 3. Field amplitudes at boundaries
And the field amplitudes at the interfaces are written as $a_{1}{ }^{+}(0)=\Gamma a_{1}{ }^{-}(0), \quad a_{1}{ }^{-}(L)=\Gamma a_{1}{ }^{+}(L)$, and $a_{2}{ }^{-}(L)=0$. Here, $\Gamma$ is a reflection coefficient which is calculated by assuming the plane wave reflection at the boundaries between the cavity and the metal:

$$
\Gamma=\frac{\beta-n_{2} k_{0}}{\beta+n_{2} k_{0}}
$$

where $k_{0}$ is the wavenumber of the incident light wave.

Consequently, the field amplitudes are related to each other as follows:

$$
\begin{aligned}
& \left(\begin{array}{l}
a_{1}^{+}(L) \\
a_{2}{ }^{+}(L)
\end{array}\right)=e^{-j \beta L}\left(\begin{array}{ll}
f_{11}(L) & f_{12}(L) \\
f_{21}(L) & f_{22}(L)
\end{array}\right)\left(\begin{array}{l}
a_{1}^{+}(0) \\
a_{2}^{+}(0)
\end{array}\right) \\
& \left(\begin{array}{l}
a_{1}{ }^{-}(0) \\
a_{2}{ }^{-}(0)
\end{array}\right)=e^{-j \beta L}\left(\begin{array}{ll}
f_{11}(L) & f_{12}(L) \\
f_{21}(L) & f_{22}(L)
\end{array}\right)\left(\begin{array}{l}
a_{1}{ }^{-}(L) \\
a_{2}{ }^{-}(L)
\end{array}\right) \\
& \left(\begin{array}{c}
\frac{1}{\Gamma} a_{1}{ }^{+}(0) \\
a_{2}{ }^{-}(0)
\end{array}\right)=e^{-j \beta L}\left(\begin{array}{cc}
f_{11}(L) & f_{12}(L) \\
f_{21}(L) & f_{22}(L)
\end{array}\right)\left(\begin{array}{c}
\Gamma a_{1}{ }^{+}(L) \\
0
\end{array}\right) .
\end{aligned}
$$

By solving (19) and (20), $a_{2}{ }^{+}(L)$ is expressed by $a_{2}{ }^{+}(0)$ as

$$
a_{2}^{+}(L)=\frac{1}{\Delta}\left[f_{22}(L)-f_{11}(L) e^{-j 2 \beta L}\right] e^{-j \beta L} a_{2}^{+}(0)
$$

where $\Delta$ is written as $\Delta=\left(1 / \Gamma^{2}\right)-f_{11}{ }^{2}(L) e^{-j 2 \beta L}$.

The power transmittance $|\mathrm{T}|^{2}$ is defined by using $a_{2}{ }^{+}(L)$ and $a_{2}{ }^{+}(0)$ as follows:

$$
|T|^{2}=\left|\frac{a_{2}{ }^{+}(L)}{a_{2}{ }^{+}(0)}\right|^{2}
$$

By substituting (21) into (22), the power transmittance is calculated as

$$
\begin{gathered}
|T|^{2}=\frac{\cos ^{2} c L\left|1-\Gamma^{2} e^{-j 2 \beta L}\right|^{2}}{\left|1-\Gamma^{2} \cos ^{2} c L e^{-j 2 \beta L}\right|^{2}} \\
=\frac{\cos ^{2} c L\left|1-\left(\Gamma_{r}+j \Gamma_{i}\right)(\cos 2 \beta L+j \sin 2 \beta L)\right|^{2}}{\left|1-\left(\Gamma_{r}+j \Gamma_{i}\right) \cos ^{2} c L(\cos 2 \beta L+j \sin 2 \beta L)\right|^{2}}
\end{gathered}
$$

$\frac{\cos ^{2} c L\left\{\left(1-\Gamma_{r} \cos 2 \beta L-\Gamma_{i} \sin 2 \beta L\right)^{2}+\left(\Gamma_{r} \sin 2 \beta L-\Gamma_{i} \cos 2 \beta L\right)^{2}\right\}}{\left(1-\Gamma_{r} \cos ^{2} c L \cos 2 \beta L-\Gamma_{i} \cos ^{2} c L \sin 2 \beta L\right)^{2}+\left(\Gamma_{r} \cos ^{2} c L \sin 2 \beta L-\Gamma_{i} \cos ^{2} c L \cos 2 \beta L\right)^{2}}=$

where $\Gamma^{2}$ is calculated using the effective refractive index $n_{e}=\beta / k_{0}$ and the imaginary part of the refractive index of the metal $\kappa=\operatorname{imag}\left(n_{2}\right)$ as

$$
\begin{gathered}
\Gamma^{2}=\frac{\left(n_{e}{ }^{2}-\kappa^{2}\right)^{2}-4 n_{e}{ }^{2} \kappa^{2}+j 4 n_{e} \kappa\left(n_{e}{ }^{2}-\kappa^{2}\right)}{\left(n_{e}{ }^{2}+\kappa^{2}\right)^{2}} \\
\triangleq \Gamma_{r}+j \Gamma_{i} .
\end{gathered}
$$

Figure 4 shows the power transmittance as a function of the incident wavelength. The results are obtained from the coupled mode-analysis and the FDTD simulations, respectively. Here, the structural parameters are chosen as follows: $a=25[\mathrm{~nm}], D=80[\mathrm{~nm}]$. It is understood from this figure that the bandstop filtering characteristics, which have several stopband frequencies, can be obtained. It is also confirmed that the results which are obtained from the coupled-mode analysis and the FDTD simulations show a good agreement with each other.

Finally, the standing wave distributions ( $\left.\left|H_{y}\right|\right)$ at the stopband wavelengths in Figs. 4(a) and (b) are shown in Figs.5 $(L=600 \mathrm{~nm})$ and $6(L=750 \mathrm{~nm})$, respectively. These 
results are obtained from the FDTD simulations. When $\lambda=450 \mathrm{~nm}(L=600 \mathrm{~nm})$, the guide wavelength $\lambda_{g}=\beta /$ $2 \pi=303 \mathrm{~nm}$, therefore, $L$ equals about $2 \lambda_{g}$. Similarly, when $\lambda=600 \mathrm{~nm}$ and $900 \mathrm{~nm}, L$ is equal to about $1.5 \lambda_{g}$ and $\lambda_{g}$, respectively. When $L=750 \mathrm{~nm}$, the standing wave distributions similar to when $L=600 \mathrm{~nm}$ are observed as shown in Fig.6, and $L$ equals about $2 \lambda_{g}, 1.5 \lambda_{g}$ and $\lambda_{g}$, respectively, when $\lambda=550 \mathrm{~nm}, 750 \mathrm{~nm}$, and $1100 \mathrm{~nm}$. It is confirmed from Figs. 5 and 6 that the stopband is shifted toward longer wavelengths as the resonator length becomes longer.

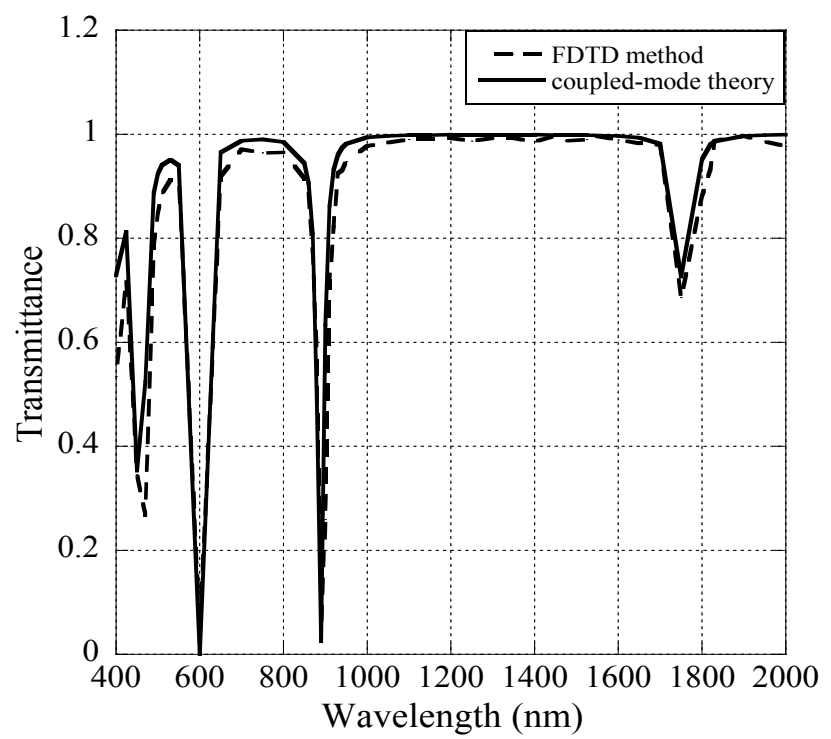

(a)

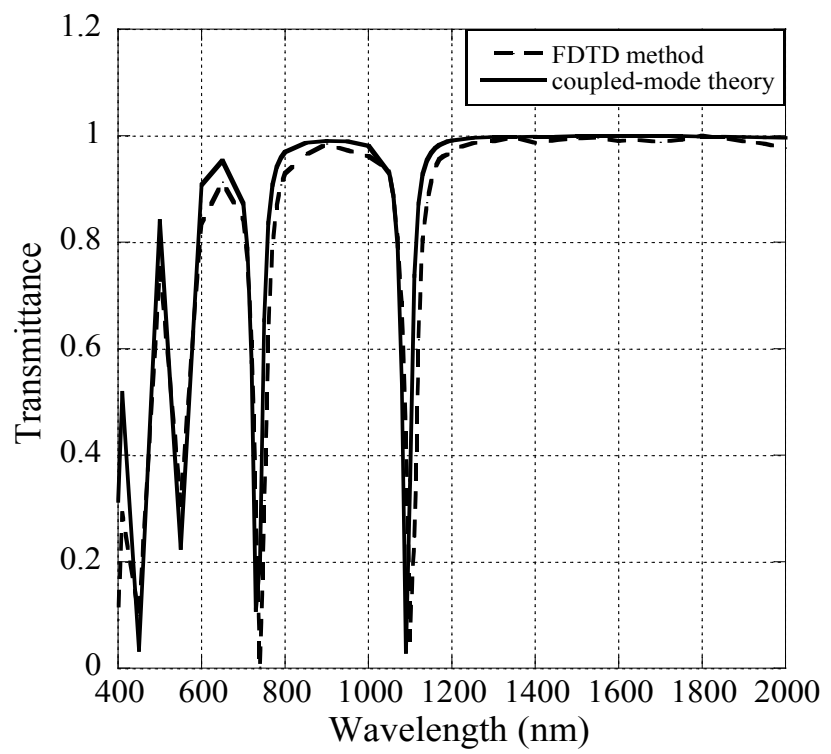

(b)

Figure 4. Power transmittance as a function of incident wavelength when (a) $L=600 \mathrm{~nm}$ and (b) $L=750 \mathrm{~nm}$

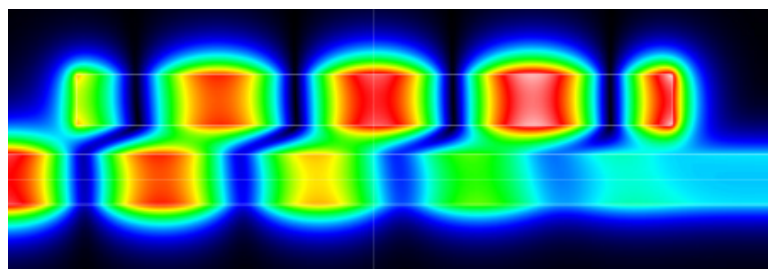

(a)

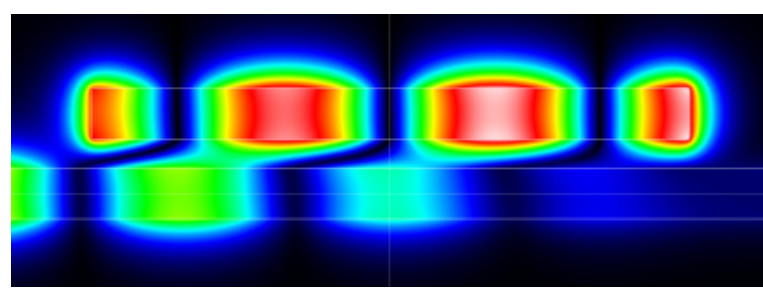

(b)

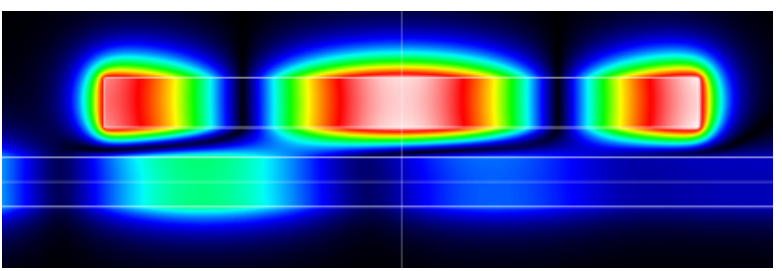

(c)

Figure 5. Standing wave distributions when (a) $\lambda=450 \mathrm{~nm}$, (b) $\lambda=600 \mathrm{~nm}$, and (c) $\lambda=900 \mathrm{~nm}$, where $L=600 \mathrm{~nm}$

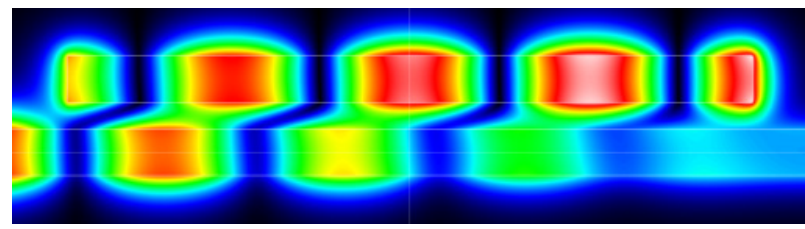

(a)

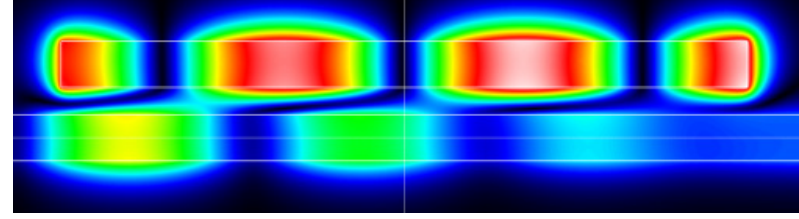

(b)

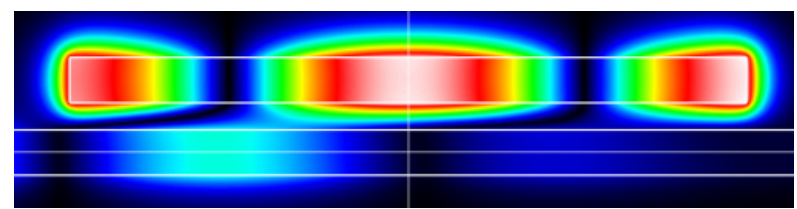

(c)

Figure 6. Standing wave distributions when (a) $\lambda=550 \mathrm{~nm}$, (b) $\lambda=750 \mathrm{~nm}$ and (c) $\lambda=1100 \mathrm{~nm}$, where $L=750 \mathrm{~nm}$

\section{Conclusions}

A resonant cavity coupled with a MIM waveguide is analyzed by using the coupled-mode theory. The coupling coefficients of two parallel MIM waveguides are analyzed utilizing the coupled-mode equations. The frequency characteristics of the power transmittance of a MIM waveguide coupled with a resonant cavity are investigated and compared with the FDTD simulation results.

\section{REFERENCES}

[1] Y. J. Chang and G. Y. Lo, "A narrowband metal-insulator-metal waveguide plasmonic Bragg grating," IEEE Photon. Technol. Lett., vol. 22, no. 9, pp.634-636, May 2010. 
[2] S. Zou, F. Wang, R. Liang, L. Xiao, and M. Hu, "A nanoscale refractive index sensor based on asymmetric plasmonic waveguide with a ring resonator: a review," IEEE Sensors J., vol. 15, no. 2, pp.646-650, Feb. 2015.

[3] Y. Chang and C. H. Chen, "Broadband plasmonic bandstop filters with a single rectangular ring resonator," IEEE Photon. Technol. Lett., vol. 26, no. 19, pp.1960-1963, Oct. 2014.

[4] J. Chen, Y. Li, Z. Chen, J. Peng, J. Qian, J. Xu, and Q. Sun, "Tunable resonances in the plasmonic split-ring resonator," IEEE Photon. J., vol. 6, no. 3, 4800706, June 2014.

[5] K. Wen, Y. Hu, L. Chen, J. Zhou, L. Lei, and Z. Guo, "Design of an optical power and wavelength splitter based on subwavelength waveguides," J. Lightwave Technol., vol. 32, no. 17 , pp. 3020-3026, Sept. 2014.

[6] M. A. Ayad, S. A. Obayya, and M. A. Swillam, "Submicron $1 \mathrm{xN}$ ultra wideband MIM plasmonic power splitters," J. Lightwave Technol., vol. 32, no. 9, pp.1814-1820, May 2014.

[7] F. Lu, Z. Wang, K. Li, and A. Xu, "A plasmonic triple-wavelength demultiplexing structure based on a MIM waveguide with side-coupled nanodisk cavities," IEEE Trans. Nanotechnol., vol.12, no. 6, pp.1185-1190, Nov. 2013.

[8] A. Pannipitiya, I. D. Rukhlenko, and M. Premaratne, "Analytical modeling of resonant cavities for plasmonic-slot-waveguide junctions," IEEE Photon. J., vol. 3, no. 2, pp. 220-233, Apr. 2011.

[9] K. H. Wen, L. S. Yan, W. Pan, B. Luo, Z. Guo, Y. H. Guo, and X. G. Luo, "Spectral characteristics of plasmonic metal-insulator-metal waveguides with a tilted groove," IEEE Photon. J., vol. 4, no. 5, pp. 1794-1800, Oct. 2012.
[10] L. O. Diniz, F. D. Nunes, E. Marega, Jr., J. Weiner, and B. H. V. Borges, "Metal-insulator-metal surfaces plasmon polariton waveguide filters with cascated transverse cavities," J. Lightwave Technol., vol. 29, no.5, Mar. 2011.

[11] J. H. Zhu, Q. J. Wang, P. Shum, and X. G. Huang, “A simple nanometric plasmonic narrow-band filter structure based on metal-insulator-metal waveguide," IEEE Trans. Nanotechnol., vol. 10, no. 6, pp. 1371-1376, Nov. 2011.

[12] J. H. Zhu, Q. J. Wang, P. Shum, and X. G. Huang, "A nanoplasmonic high-pass wavelength filter based on a metal-insulator-metal circuitous waveguide," IEEE Trans. Nanotechnol., vol. 10, no. 6. pp. 1357-1361, Nov. 2011.

[13] L. Xu, S. Wang, and L. Wu, "Refractive index sensing based on plasmonic waveguide side coupled with bilaterally located double cavities," IEEE Trans. Nanotechnol., vol. 13, no. 5, pp. 875-880, Sept. 2014.

[14] K. Kishioka, "A design method to achieve wide wavelength-flattered responses in the directional coupler-type optical power splitters," J. Lightwave Technol., vol. 19, no. 11, pp.1705-1715, Nov. 2001.

[15] K. Kishioka, "Characteristics of the optical resonator composed of the nonlinear directional coupler," IEEJ Trans. FM., vol.123, no.12, pp1166-1173, 2003.

[16] T. Kitamura, "FDTD analysis of a near-field optical disk with a ridged-square nano-aperture," IEICE Trans. Electron., vol. E95-C, no. 6, pp.1110-1116, June 2012.

[17] D. Marcuse, Light Transmission Optics, chapter 10, Van Nostrand Reinhold, New York, 1972. 